\title{
Transmission of lateral vibrations to children during car traveling and possibilities for their reduction
}

\author{
Nikolay Pavlov, ${ }^{1, *}$ Evgeni Sokolov ${ }^{1}$, Emil Iontchev ${ }^{2}$, Rossen Miletiev ${ }^{3}$, and Mihail Peychev ${ }^{1}$ \\ ${ }^{1}$ Technical University of Sofia, Faculty of Transport, 8 Kliment Ohridski Blvd., 1000 Sofia, Bulgaria \\ ${ }^{2}$ University of Transport "T. Kableshkov", Faculty of Telecommunications and Electrical Equipment in Transport, \\ 158 Geo Milev Str., 1574 Sofia, Bulgaria \\ ${ }^{3}$ Technical University of Sofia, Faculty of Telecommunication, 8 Kliment Ohridski Blvd., 1000 Sofia, Bulgaria
}

\begin{abstract}
The publication addresses the need for technical solutions to reduce the transmission of lateral vibrations to passengers, especially children traveling by car in a child seat. A special design of a pendulum type tilting child seat has been proposed. Results of road trial conducted on a specific mountain road section are shown. The differences in the values of lateral accelerations acting on a child traveling in a conventional and in a tilting seat are given. The power spectral densities of lateral accelerations for both types of child seats are presented and compared. The root mean square values of lateral acceleration are given. Results for the values of the seat tilt angle are presented.
\end{abstract}

\section{Introduction}

When vehicles move, accelerations occur and aggravate the comfort of passengers. The efforts to improve the comfort in vehicles so far are directed mainly towards reducing the amplitude of vertical oscillations by elastic suspension. In the nature of human being is to least sense the vibrations with frequencies around 1-2 Hz, corresponding to frequencies generated during walking [1]. The characteristics of suspension elements are selected so as to obtain a significant reduction in the amplitude of vertical oscillations in the frequency range 0 to $1 \mathrm{~Hz}$, and from 2 to $20 \mathrm{~Hz}$ and above. This is achieved by setting the natural frequencies of the vehicle body in the range 1-2 $\mathrm{Hz}$. Longitudinal accelerations depend mainly on acceleration and deceleration of the vehicle and do not change frequently when traveling. When vehicles move in a turn, lateral accelerations act on passengers. Their values depend on the curve radius and the vehicle speed. During car travel, usually the speeds in cornering are at discretion of the driver, according to his capabilities. On a mountain road with successive curves this gives rise to a vibrational process in the lateral direction with a frequency depending on the turning radius, length, direction, spacing and speed of the car. On roads with many turns this vibrational process usually is in the frequency domain between 0,1 and $1 \mathrm{~Hz}$ and therefore can cause discomfort and motion sickness in passengers.

To reduce the influence of lateral accelerations and to increase passenger comfort at high speeds, trains have been long using body tilting systems. The idea was discussed in 1938 in Germany. Experimental trains with passive and active tilt system were built in France in 1957 and in Germany in 1965. Later experimental trains with tilt systems were built and tested in Italy, Japan, Sweden, UK, Canada and Norway [2]. In the 70s a passive system - Talgo Pendular, for tilting the car-body was developed for the Spanish trains. In the meantime an active system for tilting the car body was designed and implemented in the Italian railroad vehicles Pendolino [3]. There are numerous studies on the directions and frequencies of vibrations of railway equipment, causing motion sickness, and also on the impact of velocity and acceleration of tilting trains on the probability of its occurrence. Recommendations for its limiting are given $[4,5]$. Tilting car body railway stocks currently operate in many countries. They allow movement with higher speed through curves and in this way the value of noncompensated lateral acceleration, causing fatigue of passengers, is reduced and does not exceed the values permitted by the international norms [6]. The passengers comfort increases and mechanical effects that cause motion sickness in passengers are reduced, especially in trains moving on railways with many curves.

In road transport there are few experimental developments of active suspension, tilting the car body towards the center of the turn. They are not approved because of their complexity, high cost, problems with stability and tire-road contact at high speeds. A solution much more simple is to use the principle of tilting the seats in the car. Even easier to accomplish is passive tilting due to inertial forces acting on extra mounted child seats. Taking into account the fact that children are the most vulnerable group of impacts causing motion sickness, the expected effect is not at all negligible.

The aim of this paper is to estimate the transmission of lateral vibrations to children while they travel by car on typical mountain road section with many turns and

Corresponding author: npavlov@tu-sofia.bg 
also to provide a technical solution for their reduction and to verify its effectiveness.

\section{Methodology}

The road experiment was conducted on the SofiaZheleznitsa-Sapareva banya route. This is a typical mountain road with many turns (Fig. 1). The route length is $69 \mathrm{~km}$.

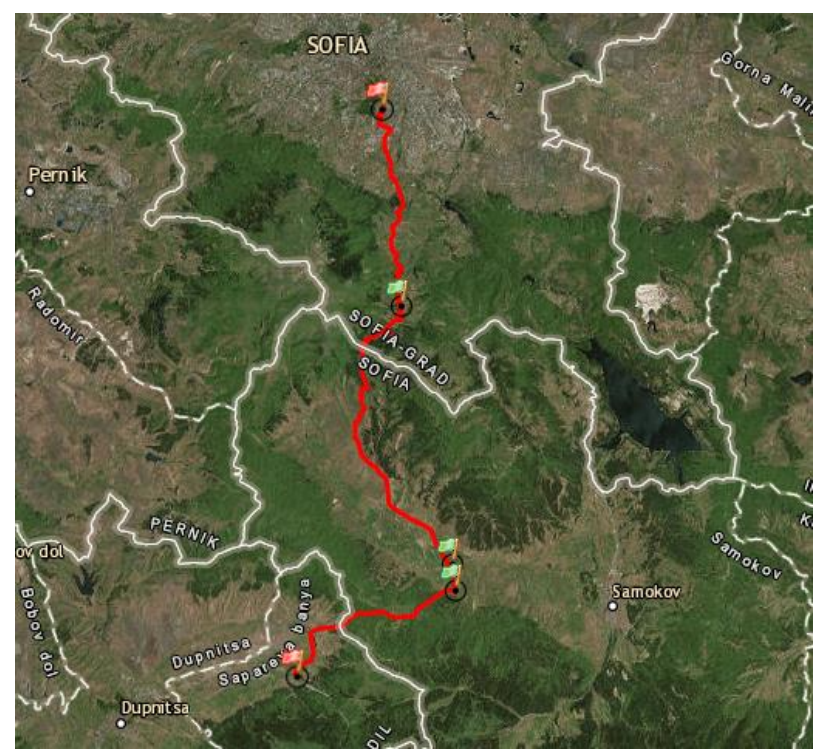

Fig. 1. Test route Sofia-Zheleznitsa-Sapareva banya [7].

Two child seats were placed in a vehicle. One of them is conventional. The other one is specially designed for the needs of the research [8]. The design of the tilting seat is shown in Fig. 2. It is child seat, which is mounted on a frame via a hinge joint in its upper end. The hinge joint is located over the mass center of the child, sitting in the seat. This way while the automobile is cornering the centrifugal force acts in the child's mass center and makes the upper part of the seat with the sitting child tilt around the joint and towards the center of the turn, while the lower part of the seat goes outwards the center of the turn [8].
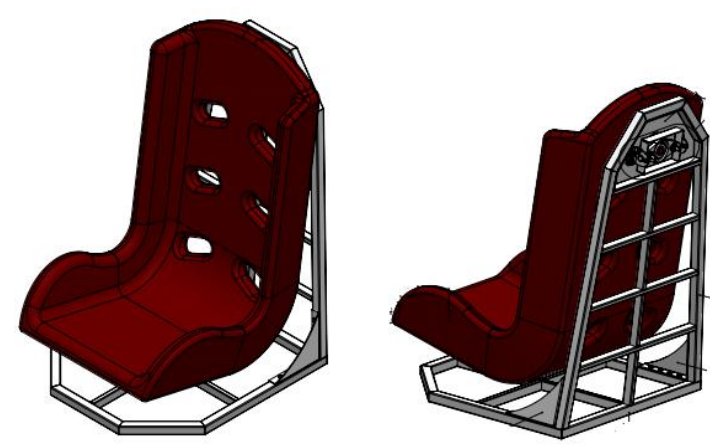

Fig. 2. Design of the tilting child seat [8].

During the trial, the accelerations data on the conventional and the tilting seat was collected. The tilt angular velocity data of the tilting child seat was collected too. An accelerometer is positioned on the conventional seat as shown in Fig. 3. An Inertial Measurement Unit including accelerometer, gyroscope and magnetometer is placed on the tilting seat instead of the accelerometer. The sensors signals were visualised and recorded on the hard disc of a mobile computer. A GPS unit is mounted in the vehicle.

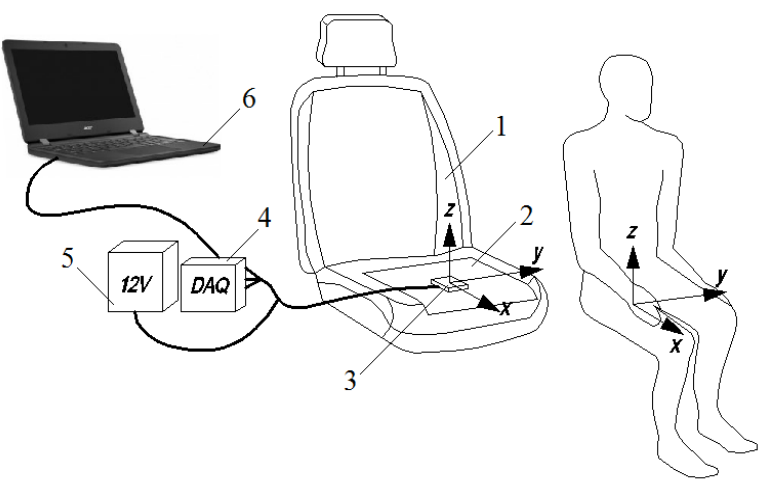

Fig. 3. Equipment, used in road trials: 1 - seat; 2 - rigid frame; 3 - inertial measurement unit (on tilting seat) or accelerometer (on conventional seat); 4 - data acquisition device; 5 - power supply; 6 - mobile computer $[8,9]$.

For safety reasons, the test is not performed with real children. It was conducted with test dummies. The mass and geometric parameters of the dummies are taken as those of real children.

\section{Results}

The data obtained from the GPS is shown in Fig. 4. It shows the trajectory of the vehicle in which the two child seats are installed - conventional and tilting.

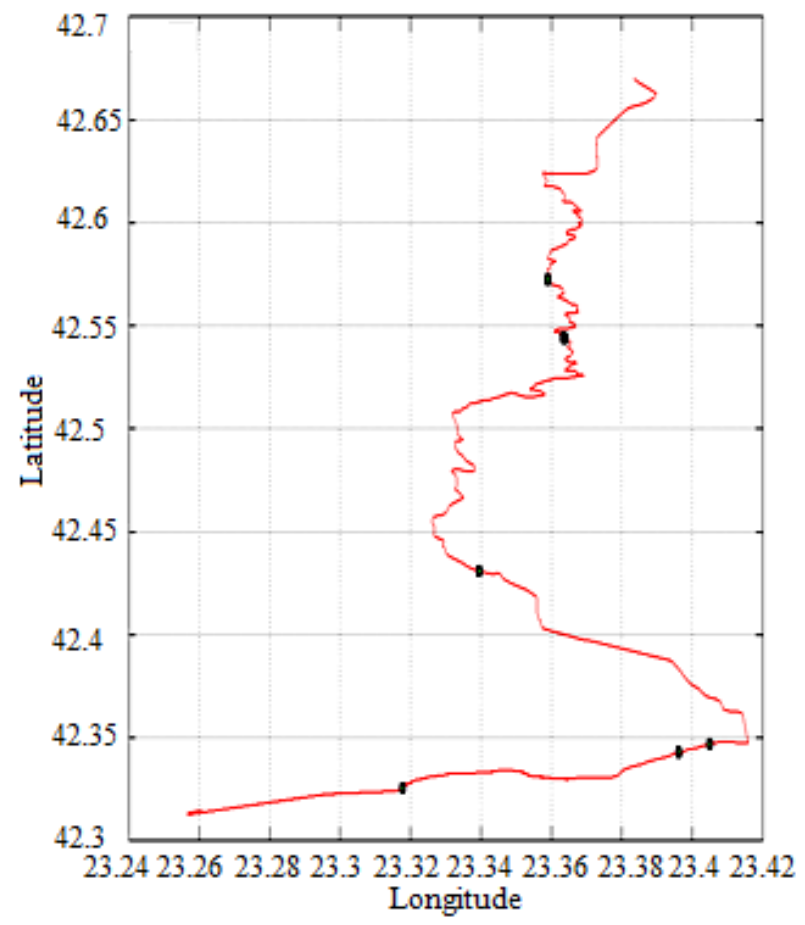

Fig. 4. Vehicle trajectory obtained from GPS. 
The lateral accelerations of conventional and tilting child seat measured and collected during the test trip are shown in Fig. 5. The results suggest that the maximum accelerations of the tilting seat are lower than the ones of the conventional seat. In both time series of the accelerations single peak values can be seen. They are probably due to passing of the vehicle through large bumps on the road and subsequent shaking of the vehicle. It may also be the case of a sudden circumvention of holes or other obstacles the road.

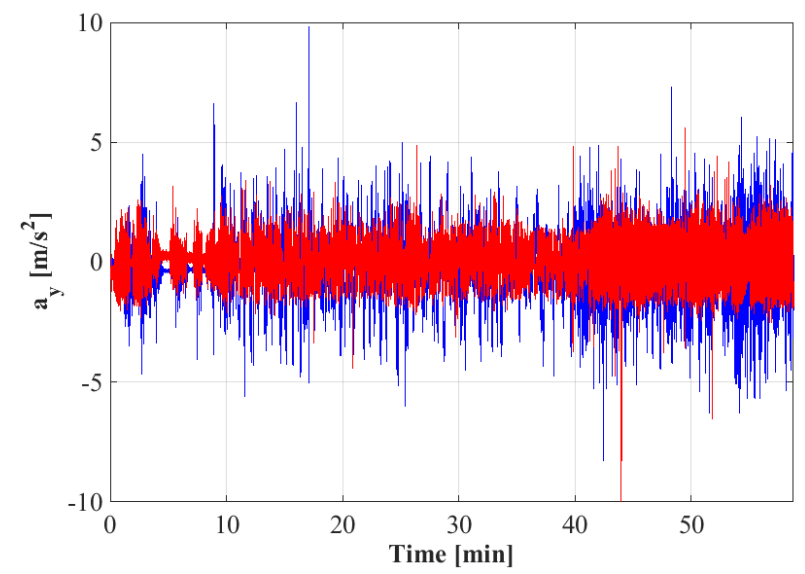

Fig. 5. Measured lateral accelerations $\left(a_{\mathrm{y}}\right)$ on conventional (blue line) and on tilting seat (red line).

The power spectral densities (PSD) of the acceleration on the both child seats are compared in Fig. 6.

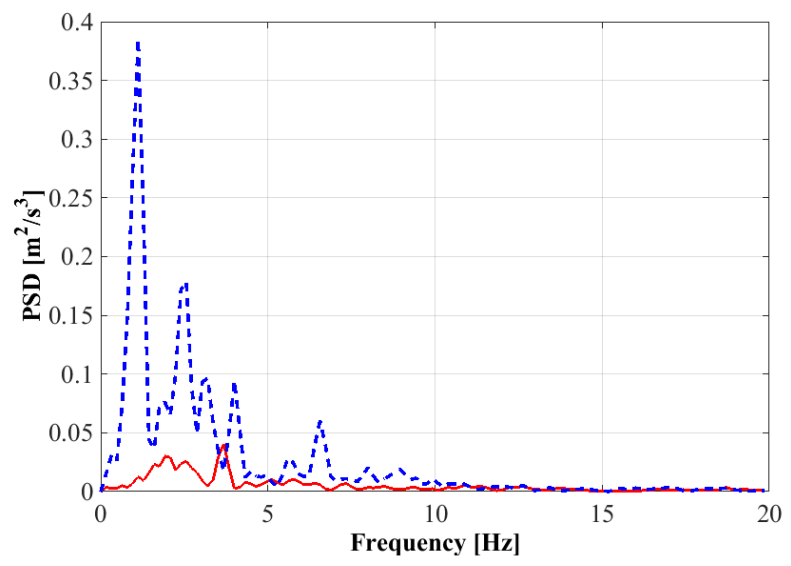

Fig. 6. Power spectral densities of the lateral accelerations on conventional (blue dotted line) and on tilting seat (red line).

This figure clearly shows that the tilting seat significantly decreases the harmful influence of lateral vibrations with frequency range from 0 to $10 \mathrm{~Hz}$, acting on the traveling child. It is particularly important for low frequencies between 0 and $1 \mathrm{~Hz}$ which cause nausea and other symptoms of motion sickness in children.

According to [10] the root mean square (RMS) value of acceleration is the basic method for vibration evaluation. It is calculated by the following formula:

$$
R M S=\left[\frac{1}{t_{D}} \int_{0}^{t_{D}} a_{w y}^{2}(t) d t\right]^{\frac{1}{2}}
$$

where $t_{D}$ is the duration of the measurement, $a_{w y}$ is the weighted lateral acceleration as a function of time in $\mathrm{m} / \mathrm{s}^{2}[10]$.

The values of the RMS of lateral acceleration for conventional child seat and for tilting child seat are:

$\mathrm{RMS}=1.12 \mathrm{~m} / \mathrm{s}^{2}$

$$
\mathrm{RMS}_{\mathrm{tilt}}=0.71 \mathrm{~m} / \mathrm{s}^{2}
$$

According to [10] the value of the conventional and the tilting seat is defined as uncomfortable and fairly uncomfortable respectively.

The difference in the RMS values of lateral acceleration confirms the efficiency of the tilting seat for reducing the lateral accelerations and improving the ride comfort.

The collected data of the gyro sensor for the angular velocity of the tilting seat is shown in Fig. 7.

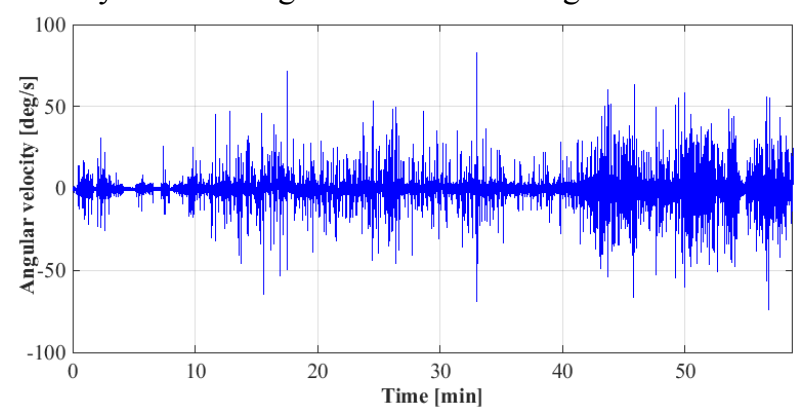

Fig. 7. Angular velocity of the tilting seat.

To estimate the seat tilt angle a Kalman filter is used [11]. The obtained results are shown in Fig. 8.

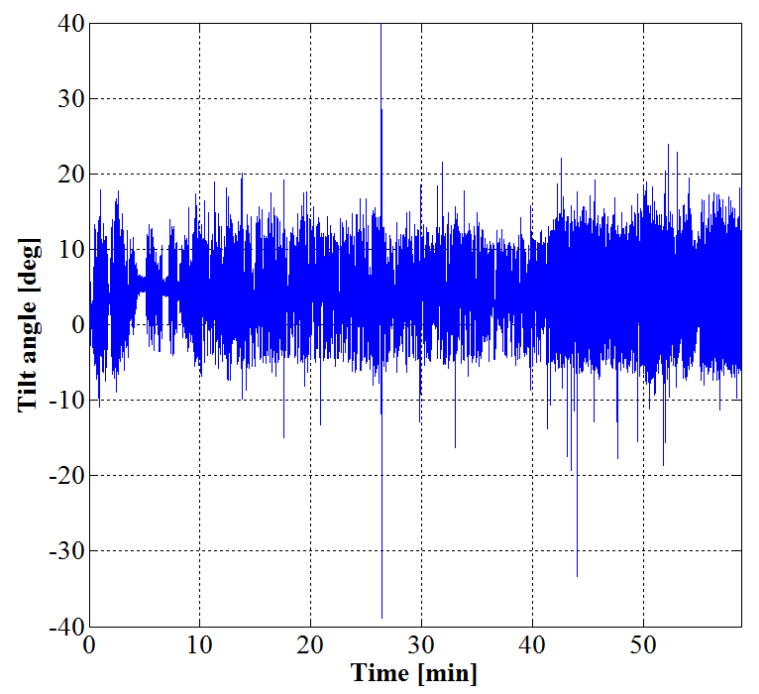

Fig. 8. Tilt angle of the tilting seat.

As can be seen from the graphs the seat tilts most often at an angle of 10-15 degrees. In some places there are also larger values, but they are probably the result of the above-mentioned singular disturbances from the road environment.

The picture during the test shows how the seat is tilted when the vehicle turns (Fig. 9). 


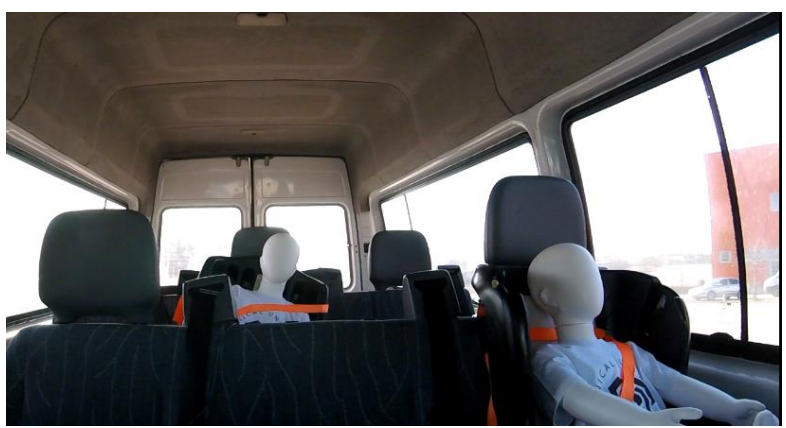

Fig. 9. View from the vehicle saloon during the test. The tilting seat is in the background.

\section{Conclusion}

A review of the state of the tilting principle solutions in ground vehicles is made in this paper. It is noted that special solutions to reduce lateral accelerations that occur in a cornering vehicle are currently not used. They act on the passengers and aggravate their comfort. A technical solution is proposed to reduce lateral accelerations that occur when driving on roads with many turns and have the nature of a low-frequency vibrational process. The solution is directed towards one of the most vulnerable groups of motion sickness children, and represents a tilting child seat of a pendulum type.

A suitable route was chosen and a road test was conducted. Results for the values of lateral accelerations acting on the conventional and on the tilting child seats were obtained during the experiment. They show a significant reduction of the lateral accelerations with the use of a tilting child seat. Results for the angular velocities of the tilting child seat were also gathered. The values of angles of tilting of the seat were also obtained after applying a procedure with the use of a Kalman filter.

This work was supported by the National Science Fund of the Ministry of Education and Science of the Republic of Bulgaria [Grant No. ДМ 07/8 (December 17, 2016)].

\section{References}

1. R. V. Rotenberg, Vehicle suspension. Vibration and ride comfort. (Mashinostroenie, Moscow, 1972)

2. J. Förstberg, Ride comfort and motion sickness in tilting trains. (Stockholm, 2000)

3. A. Steimel, Electric traction - motive power and energy supply. (Oldenbourg Industriverlag, Munich, 2008)

4. J. Förstberg, Nausea and comfort in tilting trains: Possible regression models for nausea. The Dynamics of Vehicles on Roads and on Tracks Supplement to Vehicle System Dynamics, 41, pp. 302-310, (2004)

5. T. Wiederkehr, Review of motion sickness evaluation methods and their application to simulation technology. SIMPACK News, July (2013)

6. G. Lauriks, J. Evans, J. Förstberg, M. Balli and I. Barron de Angoiti, UIC comfort tests: Investigation of ride comfort and comfort disturbance on transition and circular curves. (VTI notat 56A, 2003)

7. https://www.maps.ie/map-my-route/

8. N. Pavlov, E. Sokolov, M. Peychev, D. Dacova, Design and test of a tilting seat for improving children's comfort during traveling. ITEMA Conference proceedings (2018)

9. N. Pavlov, D. Dacova, Comparative Analysis of the Accelerations Acting on Passengers in Road and Rail Transport, Proceedings of the technical and scientific conference EKO Varna, pp. 246-252, (2017)

10. ISO 2631-1. International Organization for Standardization, Mechanical vibration and shock evaluation of human exposure to whole body vibration - part 1: General requirement, (1997)

11. E. Iontchev, R. Kenov, R. Miletiev, Inertial measurement system for evaluation of the bogierailway system dynamics, 36th International Spring Seminar on Electronics Technology, „Automotive Electronics“, Alba Iulia, Romania, pp. 172-173 (2013) 